\title{
LINE-STRENGTH INDICES IN BRIGHT SPHEROIDAL GALAXIES: EVIDENCE FOR A STELLAR POPULATION DICHOTOMY BETWEEN SPHEROIDAL AND ELLIPTICAL GALAXIES
}

\author{
Javier Gorgas, ${ }^{1}$ Santos Pedraz, ${ }^{1}$ Rafael Guzmán, ${ }^{2}$ Nicolás Cardiel, ${ }^{1}$ J. Jesús González ${ }^{3}$ \\ Received 1996 November 13; accepted 1997 March 3
}

\begin{abstract}
We present new measurements of central line-strength indices (namely, $\mathrm{Mg}_{2},\langle\mathrm{Fe}\rangle$, and $\mathrm{H} \beta$ ) and gradients for a sample of six bright spheroidal (Sph) galaxies in the Virgo Cluster. Comparison with similar measurements for elliptical (E) galaxies, galactic globular clusters (GGCs), and stellar population models yield the following results: (1) in contrast with bright $\mathrm{E}$ galaxies, bright Sph galaxies are consistent with solar abundance $[\mathrm{Mg} / \mathrm{Fe}] \mathrm{ratios}$; (2) bright Sph galaxies exhibit metallicities ranging from values typical for metal-rich GGCs to those for E galaxies; (3) although absolute mean ages are quite model dependent, we find evidence that the stellar populations of some (if not all) Sph galaxies look significantly younger than GGCs; and (4) $\mathrm{Mg}_{2}$ gradients of bright Sph galaxies are significantly shallower than those of $\mathrm{E}$ galaxies. We conclude that the dichotomy found in the structural properties of Sph and E galaxies is also observed in their stellar populations. A tentative interpretation in terms of differences in star formation histories is suggested.
\end{abstract}

Subject headings: galaxies: abundances — galaxies: evolution — galaxies: stellar content

\section{INTRODUCTION}

Over the last few years the existence of a structural dichotomy between elliptical (E) and spheroidal (Sph) galaxies, ${ }^{4}$ has been established (see, e.g., Kormendy 1985; Binggeli \& Cameron 1991), which suggests different galaxy formation/evolution processes for both kinds of galaxies (Dekel \& Silk 1986; Guzmán, Lucey, \& Bower 1993). However, this dichotomy contrasts with the remarkable similarity in the global properties of their stellar populations. In particular, the color-luminosity relation as well as the correlation between the $\mathrm{Mg}_{2}$ line-strength index and velocity dispersion are apparently universal for both galaxy families (Caldwell 1983; Bender 1991). A detailed comparison of the star formation histories of Sph and E galaxies is required to provide constraints on their formation mechanisms. Also, understanding the star formation history of Sph galaxies is of vital importance for cosmological issues such as the nature of the faint blue galaxies (Babul \& Rees 1992) or the faint end of the luminosity function.

Recent studies devoted to comparing in detail the stellar populations of Sph and E galaxies have led to ambiguous results so far. For dwarf Sph galaxies in the Local Group, there is now clear evidence of recent (i.e., 3-5 Gyr) star-forming events (see, e.g., da Costa 1991). Spectroscopic studies have provided evidence that some bright Sph galaxies do exhibit a young or intermediate-age stellar population (Gregg 1991; Held \& Mould 1994). However, the general trend is that bright Sph galaxies tend to follow the galactic globular cluster (GGC) locus in the color-line-strength diagrams, but with a large scatter (Bothun \& Mould 1988; Brodie \& Huchra 1991; Held \& Mould 1994). Ferguson (1994) has compared colors and line strengths for Sph galaxies, E galaxies, and GGCs with the

\footnotetext{
1 Departamento de Astrofísica, Facultad de Físicas, Universidad Complutense, Madrid, E28040, Spain.

2 UCO/Lick Observatory, University of California, Santa Cruz, CA 95064.

${ }^{3}$ Instituto de Astronomía, UNAM, Apdo. Postal 70-264, 04510 Mexico D.F., Mexico.

4 Throughout this Letter we adopt the nomenclature of Kormendy \& Bender (1994), i.e., low-density, dwarf ellipsoidal galaxies such as NGC 205 are called "spheroidal" instead of "dwarf elliptical," as in Binggeli (1994).
}

predictions of stellar population models, concluding that differences in the line-strength-color relations may arise because of calibration problems and the relatively large uncertainties in both the measurements and the population synthesis models. Clearly, a systematic study with more precise measurements is needed to derive any serious constraints on the stellar populations of Sph galaxies. In this Letter we show the first results of a spectroscopic survey of Sph galaxies aimed at studying in detail their stellar populations and kinematics. The analysis presented here is based on new central measurements of line-strength indices (namely, $\mathrm{Mg}_{2}, \mathrm{Fe} 5270, \mathrm{Fe} 5335$, and $\mathrm{H} \beta$ ) and, for the first time, gradients for a sample of six Sph galaxies in the Virgo Cluster.

\section{THE DATA}

The galaxy sample listed in Table 1 includes six bright Sph galaxies in the Virgo Cluster. Long-slit spectroscopic observations were carried out during 1994 April 11-15, with the $2.5 \mathrm{~m}$ Isaac Newton Telescope (INT) at La Palma. The IDS spectrograph provided $2.5 \AA$ (FWHM) resolution spectra in the wavelength range $4700-6100 \AA$. The slit was aligned with the major axis. Exposure times (typically $\sim 4 \mathrm{hr}$ per galaxy) allow measurements of spectral features out to the galaxy effective radius $r_{e}$. Spectra of the central regions have very high quality (the signal-to-noise ratio ranges from 32 to 110). A detailed description of the observational setup and reduction procedures will be given in Gorgas et al. (1997). We emphasize that, in order to ensure reliable line-strength gradients, the sky was carefully estimated at the slit ends, taking into account any possible contribution of the galaxy itself and the effect of scattered light. For each galaxy spectrum we measured the $\mathrm{Mg}_{2}, \mathrm{H} \beta, \mathrm{Fe} 5270$, and Fe5335 indices. The errors in these measurements were estimated by reducing, parallel to the galaxy frames, error images created from photon and readout noises. Since line-strength indices depend on spectral resolution, our spectra were broadened to match the resolution of the widely used Lick system $\left(200 \mathrm{~km} \mathrm{~s}^{-1}\right)$. To ensure the 
TABLE 1

Central Line Strengths

\begin{tabular}{|c|c|c|c|c|c|}
\hline Galaxy & $M_{B}^{\mathrm{a}}$ & $\sigma^{\mathrm{b}}$ & $\mathrm{Mg}_{2}$ & $\mathrm{H} \beta$ & $\langle\mathrm{Fe}\rangle^{\mathrm{c}}$ \\
\hline NGC 4415. & -17.76 & $\ldots$ & $0.182 \pm 0.005$ & $1.97 \pm 0.20$ & $2.35 \pm 0.13$ \\
\hline NGC $4431 \ldots . . . \ldots$. & -17.99 & 68 & $0.184 \pm 0.005$ & $1.88 \pm 0.20$ & $2.15 \pm 0.14$ \\
\hline NGC $4489 \ldots \ldots \ldots$. & -18.76 & 48 & $0.213 \pm 0.002$ & $2.40 \pm 0.08$ & $2.77 \pm 0.06$ \\
\hline IC $794 \ldots \ldots \ldots \ldots$ & -17.42 & 54 & $0.212 \pm 0.005$ & $2.11 \pm 0.22$ & $2.72 \pm 0.14$ \\
\hline IC $3393 \ldots \ldots \ldots \ldots$ & -17.07 & 55 & $0.142 \pm 0.006$ & $2.16 \pm 0.25$ & $2.13 \pm 0.17$ \\
\hline UGC $7436 \ldots \ldots \ldots$. & -17.27 & 45 & $0.157 \pm 0.006$ & $1.90 \pm 0.27$ & $1.88 \pm 0.18$ \\
\hline
\end{tabular}

a Sources for $B_{T}$ are Binggeli, Sandage, \& Tammann 1985 and Binggeli \& Cameron 1991. A distance to Virgo of 20.7 Mpc has been adopted (Bender, Burstein, \& Faber 1992).

${ }^{\mathrm{b}}$ Central velocity dispersion, in $\mathrm{km} \mathrm{s}^{-1}$, from Bender et al. 1992 and G93.

${ }^{c}\langle\mathrm{Fe}\rangle$ is defined as $(\mathrm{Fe} 5270+\mathrm{Fe} 5335) / 2$.

accuracy of this correction and check for any systematic errors, we observed a sample of 39 F-M stars from the Lick stellar library (Gorgas et al. 1993). After the broadening correction, we found no systematic deviations for the $\mathrm{Fe}$ and $\mathrm{H} \beta$ indices between both data sets. The $\mathrm{Mg}_{2}$ index, however, shows a systematic offset of $0.013 \mathrm{mag}$ (our values below those of Lick), which was applied to convert our indices to the Lick system.

\section{CENTRAL LINE-STRENGTH INDICES}

In Table 1 we list, for our galaxy sample, the central $\mathrm{Mg}_{2}$, $\mathrm{H} \beta$, and $\langle\mathrm{Fe}\rangle$ line-strength indices and their formal errors in a $2^{\prime \prime} \times 4^{\prime \prime}$ aperture centered on the galaxy nucleus. In Figure 1 we present line-strength diagrams for our sample of Sph galaxies as well as for a representative sample of GGCs and E galaxies. We also show the predictions of single-burst stellar population models of a given age and metallicity (Worthey 1994). It is immediately apparent that, in the $\mathrm{Mg}_{2}-\langle\mathrm{Fe}\rangle$ plane, bright $\mathrm{Sph}$ galaxies do not follow the extrapolation of the $\mathrm{E}$ sequence toward lower $\mathrm{Mg}_{2}$ values, but tend to resemble metal-rich GGCs.

The failure to reproduce theoretically the high $\mathrm{Mg}_{2}$ values observed in bright $\mathrm{E}$ galaxies has been interpreted to be due to an enhancement of the $[\mathrm{Mg} / \mathrm{Fe}]$ ratio in these galaxies relative to the solar ratio assumed in the population models (Peletier 1989; Worthey, Faber, \& González 1992; Davies, Sadler, \& Peletier 1993, hereafter DSP). From Figure 1a, it is clear that the model lines pass through the $\mathrm{Sph}$ galaxies locus, thus suggesting solar abundance $[\mathrm{Mg} / \mathrm{Fe}]$ ratios for these galaxies. This difference in $[\mathrm{Mg} / \mathrm{Fe}]$ between $\mathrm{E}$ galaxies and $\mathrm{Sph}$ galaxies may likely imply different star formation histories for both galaxy types. If the enhancement of $[\mathrm{Mg} / \mathrm{Fe}]$ is due to an overabundance of light elements in bright $\mathrm{E}$ galaxies, then it is plausible that the star formation in these galaxies occurred in a short timescale, since $\mathrm{Mg}$ is created rapidly by Type II supernovae, while $\mathrm{Fe}$ is produced in a longer timescale by Type Ia supernovae. Under this assumption, our observations imply a longer star formation period in Sph galaxies as compared to E galaxies. In other words, the star-forming event should have elapsed long enough to yield a solar $[\mathrm{Mg} / \mathrm{Fe}]$ ratio. However, other factors, such as a flatter initial mass function (IMF) in giant $\mathrm{E}$ galaxies compared to $\mathrm{Sph}$ galaxies, could account for the $\mathrm{Mg}$ overabundance difference (Worthey et al. 1992).

The positions of the bright $\mathrm{Sph}$ galaxies in the $\langle\mathrm{Fe}\rangle-\mathrm{H} \beta$ diagram also reveal a clear dichotomy in the way $\mathrm{Sph}$ and $\mathrm{E}$ galaxies populate the age-metallicity plane (Fig. 1b). Compar- ison between the measured indices and the spectral synthesis models in this diagram allows us to break the degeneracy between age and metallicity present in Figure $1 a$. Within the errors, bright Sph galaxies exhibit a large range of metallicities, from values typical for metal-rich GGCs $([\mathrm{Fe} / \mathrm{H}] \sim-0.75)$ to those for E galaxies. We note that the derived metallicities for Sph galaxies are the same and lower than for E galaxies, whether computed from $\mathrm{Mg}$ or Fe lines.

Concerning the age question, we find that bright Sph galaxies span a wide range in mean stellar ages, showing, in fact, a comparable age spread to that derived, using a similar diagram, by González (1993, hereafter G93) for E galaxies. Most interestingly, we find a trend between age and metallicity, in the sense that "younger" Sph galaxies tend to be more metal rich, although the sample is too small to reach a firm conclusion. It is important to note that the computed ages are light-weighted mean stellar ages. Moreover, since single-burst models are probably a naive approximation to the star-forming history of early-type galaxies, and given the discrepancies in the derived ages when using different models (typically $\sim 4 \mathrm{Gyr}$ for old populations and metallicities around solar; see Worthey 1994; Vazdekis et al. 1996; Fig. 1b), it is hard to give any reliable estimate of absolute ages, but the relative trends remain. Perhaps the key question is whether some $\mathrm{Sph}$ galaxies (such as UGC 7436 and NGC 4431) are as old as the oldest E galaxies. Figure $1 b$ suggests that this is the case, but this conclusion may be subject to other effects. Since the slope of the constant-age lines (for old stellar populations) in the $\langle\mathrm{Fe}\rangle-\mathrm{H} \beta$ plane is not model dependent, it is hard to reconcile the relative positions of the low- $\mathrm{H} \beta \mathrm{E}$ galaxies and metal-rich GGCs in this diagram with the idea that both are old and coeval. Vazdekis et al. (1996) have shown that the location of those constant-age lines depends on the adopted IMF slope (see Fig. 1b). Therefore, the assumption of a flatter IMF for the bright E galaxies would help to explain the position of both populations (GGCs and low- $\mathrm{H} \beta \mathrm{E}$ galaxies) in this diagram without introducing important age differences, accounting at the same time for the $\mathrm{Mg}$ overabundance effect. Under this view, the stellar populations of all bright Sph galaxies of the sample (with solar $\mathrm{Mg} / \mathrm{Fe}$ ratios) could be significantly younger than GGCs and, therefore, than the oldest bright $\mathrm{E}$ galaxies.

An important question raised by Ferguson \& Binggeli (1994) is whether the stellar populations of Sph galaxies resemble those in the outer regions of $E$ galaxies. Using line-strength profiles from G93, when the comparison is made at constant surface brightness $\left(\mu_{B}=20-21 \mathrm{mag} \operatorname{arcsec}^{-2}\right.$, 

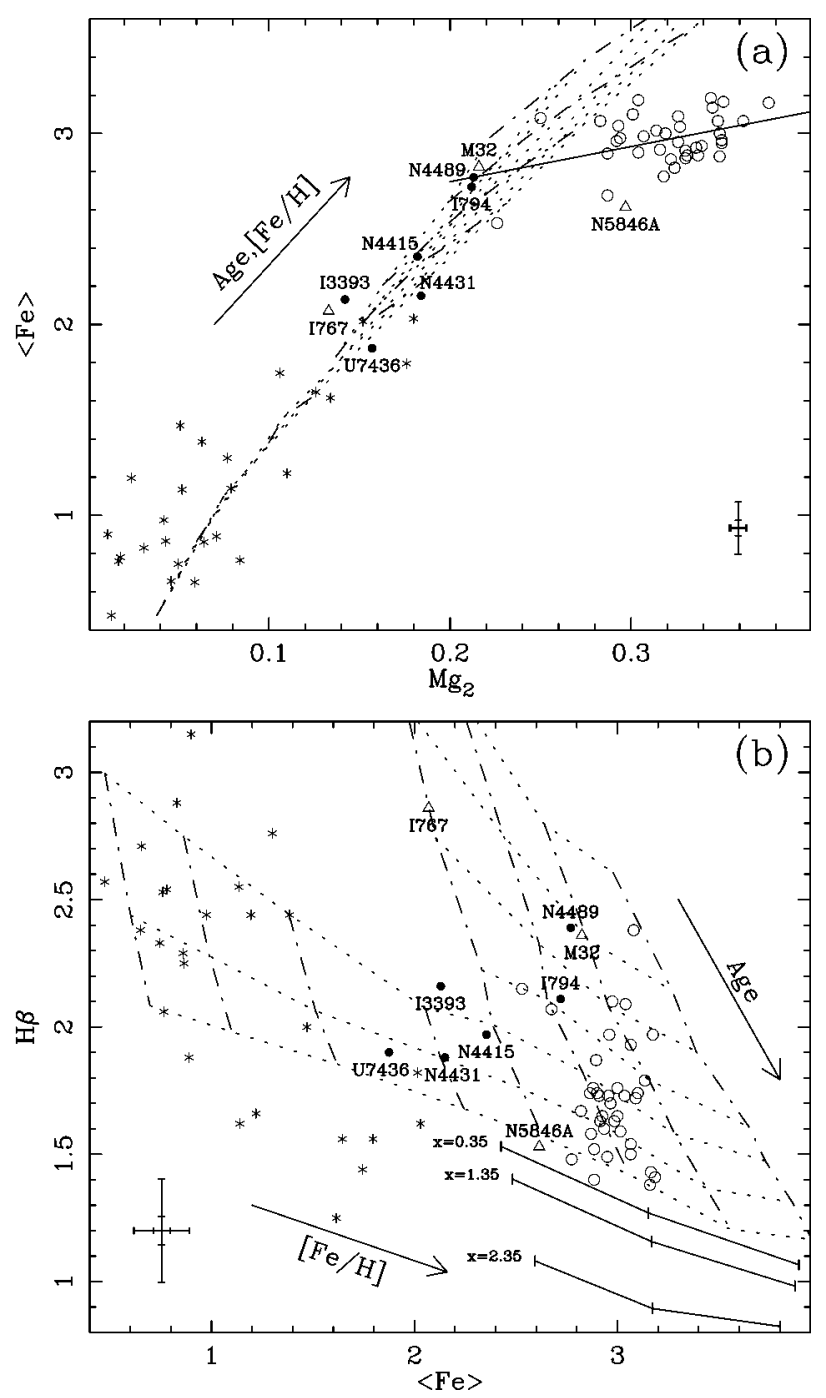

FIG. 1.-Line-strength diagrams for GGCs (asterisks, from Burstein et al. 1984, and Covino, Galletti, \& Pasinetti 1995), E galaxies (open circles, from G93), and the central regions of bright Sph galaxies (filled circles). Open triangles represent compact E galaxies from G93 (M32) and Gorgas et al. (1997) (NGC 5846A and IC 767). The straight line in $(a)$ is a least-squares fit to the G93 sample of E galaxies. Predictions from stellar population models (Worthey 1994) are shown as dotted lines (for fixed ages of 1.5, 2, 3, 5, 8, 12, and $17 \mathrm{Gyr}$, from top to bottom in $b$ ) and dot-dashed lines (for fixed metallicities of $[\mathrm{Fe} / \mathrm{H}]=-2.0,-1.5,-1.0,-0.5,-0.25,0.0,0.25,0.50$, from left to right in $b$ ). These lines overlap in $(a)$. Full lines in $(b)$ represent the predictions of single-burst models from Vazdekis et al. (1996) for a $17 \mathrm{Gyr}$ old stellar population, metallicities $[\mathrm{Fe} / \mathrm{H}]=-0.4,0,0.4$ (from left to right), and different IMF slopes $(x=1.35$ corresponds to the Salpeter $1955 \mathrm{IMF}$, and it is the slope used in Worthey models). The discrepancy between both sets of models is mainly due to differences in the temperatures of the adopted isochrones. Error bars show the typical observational errors in the indices of Sph galaxies (large crosses) and E galaxies (small crosses).

corresponding to the Sph mean brightness inside our central aperture), E galaxies attain typical $\mathrm{Mg}_{2}$ line strengths of about $0.28 \mathrm{mag}$, which are significantly larger than those observed for Sph galaxies (see Table 1). Therefore, the stellar populations are, again, different, and local surface brightness does not seem to be a main parameter in fixing the properties of the stellar populations, suggesting that local stellar populations mostly reflect the global properties (e.g., mass) and only secondarily the local environment.

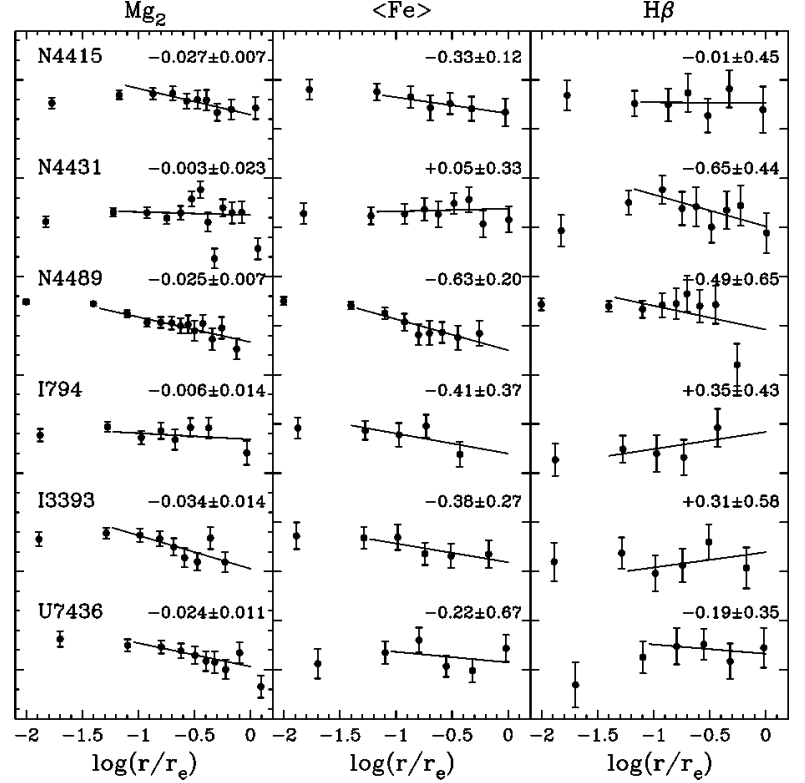

FIG. 2.- $\mathrm{Mg}_{2},\langle\mathrm{Fe}\rangle$, and $\mathrm{H} \beta$ gradients for our sample of bright Sph galaxies. Straight lines represent error-weighted least-squares fits to points between 1."5 and the effective radius $\left(r_{e}\right)$. For each galaxy we give the derived gradients and their formal errors. The individual profiles have been shifted vertically by arbitrary amounts; tick marks on the ordinate axis correspond to 0.02 mag for $\mathrm{Mg}_{2}$, and $1 \AA$ for the atomic indices $(\langle\mathrm{Fe}\rangle$ and $\mathrm{H} \beta)$. The central indices listed in Table 1 correspond to the added spectra of the two innermost points in the line-strength profiles.

\section{LINE-STRENGTH GRADIENTS}

Radial profiles of $\mathrm{Mg}_{2},\langle\mathrm{Fe}\rangle$, and $\mathrm{H} \beta$ for our galaxy sample are plotted in Figure 2. Each point along the radius corresponds to an average of line strengths from symmetrical bins at both sides of the galaxy. For the outer regions, we co-added a sufficient number of spectra in the spatial direction to guarantee a minimum signal-to-noise ratio per angstrom, leading to typical errors outside the centers of $\Delta \mathrm{Mg}_{2}=0.011 \mathrm{mag}$, $\Delta\langle\mathrm{Fe}\rangle=0.24 \AA$, and $\Delta \mathrm{H} \beta=0.36 \AA$. The gradients have been estimated from the error-weighted linear regression fits over the radial range covering from 1.5 (in order to avoid seeing effects) to the effective radius.

As is apparent from Figure 2, bright Sph galaxies possess shallow $\mathrm{Mg}_{2}$ gradients. The mean $\mathrm{Mg}_{2}$ gradient $\left(\left\langle d \mathrm{Mg}_{2} / d\right.\right.$ $\log r\rangle)$ for our sample is -0.020 , with an rms dispersion around the mean of 0.012 . This value can be compared with the mean $\mathrm{Mg}_{2}$ gradient derived by González \& Gorgas (1997) using published and unpublished data for 109 early-type galaxies with reliable $\mathrm{Mg}_{2}$ profiles. Following a fitting procedure similar to that described above, the derived mean $\mathrm{Mg}_{2}$ gradient for $\mathrm{E}$ galaxies is -0.055 , with a scatter of 0.025 . Applying the Mann-Whitney $U$-test, we conclude that $\mathrm{Mg}_{2}$ gradients in bright $\mathrm{Sph}$ galaxies are flatter than those in E galaxies at the 0.0005 level of confidence. Because of their dependence on spectral resolution and photon noise, $\mathrm{Fe}$ indices are worse determined than $\mathrm{Mg}_{2}$. Nevertheless, Fe gradients in bright $\mathrm{Sph}$ galaxies are also found to be moderately shallow. The mean $\langle\mathrm{Fe}\rangle$ gradient for the sample is -0.32 , with a scatter of 0.23 . Combining data from Gorgas, Efstathiou, \& AragónSalamanca (1990), DSP, and G93, we have derived a mean $\langle\mathrm{Fe}\rangle$ gradient for a sample of $50 \mathrm{E}$ galaxies of -0.43 (with a scatter of 0.29 ). This is also steeper than the gradients in our 
sample of Sph galaxies, although at a confidence level of only 0.26 .

Concerning $\mathrm{H} \beta$ gradients, it is clear from Figure 2 that almost all bright $\mathrm{Sph}$ galaxies in our sample exhibit essentially flat gradients in the $\mathrm{H} \beta$ strength. $\mathrm{H} \beta$ line strengths could be affected by filling due to weak nebular emission. It must be noted, however, that no [O III] $\lambda 5007$ emission lines are detectable in the spectra of these galaxies. For our sample, we derive $\langle d H \beta / d \log r\rangle=-0.11$, with an rms scatter of 0.41 , consistent with a flat mean gradient. This result agrees with what has been found for this feature in several samples of $\mathrm{E}$ galaxies (Gorgas et al. 1990; DSP; G93).

These line-strength gradients should be interpreted in terms of radial variations in mean age and metallicity. If we assumed that $\mathrm{Mg}_{2}$ gradients are entirely due to metallicity variations within galaxies, then, using Worthey (1994) models, these gradients would translate into a mean $\langle\Delta[\mathrm{Fe} / \mathrm{H}] / \Delta \log r\rangle=$ $-0.14 \pm 0.04$, considerably flatter than the mean metallicity gradient, derived in a similar way, for $\mathrm{E}$ galaxies $(-0.22$, Gorgas et al. 1990; -0.23, DSP; -0.25 , Fisher, Franx, \& Illingworth 1995). The constancy of $\mathrm{H} \beta$ along radius in galaxies with negative metallicity gradients has been interpreted, when taking the models literally, as evidence for age gradients (G93; Fisher et al. 1995). This result would also apply to our sample of Sph galaxies. Although there is a large variation from galaxy to galaxy, our data suggest moderate age gradients, in the sense of younger looking stellar populations in the inner regions relative to the outer parts. Color gradients in Sph galaxies have previously been studied by Vader at al. (1988) and Chaboyer (1994). Their main conclusion is that Sph galaxies exhibit shallower gradients than $\mathrm{E}$ galaxies, being, in the mean, compatible with a flat $B-R$ color gradient. These results are fully consistent with our line-strength gradients since age and metallicity effects would tend to cancel to yield shallow color gradients. Note that in the presence of age gradients, the above estimate for the mean metallicity gradient would be in fact underestimated (by $\sim 0.10 \mathrm{dex}$ ). In any case, since this would also apply to giant E galaxies, the metallicity gradients in Sph galaxies still remain shallower than those in $\mathrm{E}$ galaxies. This result suggests that supernova-driven winds, or some other heating mechanism, govern the chemical evolution of Sph galaxies, reducing the infall of enriched gas toward the galaxy centers and preventing, therefore, the development of steep metallicity gradients.

\section{CONCLUSIONS}

Through the study of absorption features, we present evidence that the dichotomy found between the structural properties of Sph galaxies and E galaxies (e.g., Bender, Burstein, \& Faber 1992) is also observed in the stellar populations. This dichotomy rests mainly upon the observed differences in $\mathrm{Mg} / \mathrm{Fe}$ overabundance, global metallicity, and metallicity gradients. Although, in light of the available stellar population models, these results are still unable to identify unambiguously the differences in the star formation histories of the E and Sph families, our suggestion is that, while the bulk of star formation in giant $\mathrm{E}$ galaxies occurred in a short timescale and probably with a IMF skewed toward higher masses, star formation in Sph galaxies has proceeded, self-regulated by galactic winds or other mechanisms, quietly in a longer timescale and with less amount of dissipation. Further work is needed to decide whether this elapsed star-forming period would be able to account for the young mean ages observed in some (if not all) Sph galaxies or whether subsequent starbursts should be invoked. It is clear that other important aspects, such as the possible differences between the stellar populations of nucleated and nonnucleated Sph galaxies and the influence of environment, require further investigation before we can have a more complete picture of the star formation history in spheroidal galaxies.

We are grateful to the anonymous referee, A. AragónSalamanca, J. Gallego, and R. Peletier for useful comments. The INT is operated on the island of La Palma by the RGO at the Observatorio del Roque de los Muchachos of the Instituto de Astrofísica de Canarias. This work was supported in part by the Spanish grant PB93-456. R. G. acknowledges funding from the Spanish MEC fellowship EX93-27295297 and NSF grant AST91-20005.

\section{REFERENCES}

Babul, A., \& Rees, M. 1992, MNRAS, 255, 346

Bender, R. 1991, in IAU Symp. 149, The Stellar Populations of Galaxies, ed. B. Barbuy \& A. Renzini (Dordrecht: Kluwer), 269

Bender, R., Burstein, D., \& Faber, S. M. 1992, ApJ, 399, 462

Binggeli, B. 1994, in ESO Conf. and Workshop Proc. 49, Dwarf Galaxies, ed. G.

Meylan \& P. Prugniel (Haute Provence: ESO), 13

Binggeli, B., \& Cameron, L. M. 1991, A\&A, 252, 27

Binggeli, B., Sandage, A., \& Tammann, G. A. 1985, AJ, 90, 1681

Bothun, G. D., \& Mould, J. R. 1988, ApJ, 324, 123

Brodie, J. P., \& Huchra, J. P. 1991, ApJ, 379, 157

Burstein, D., Faber, S. M., Gaskell, C. M., \& Krumm, N. 1984, ApJ, 287, 586 Caldwell, N. 1983, AJ, 88, 804

Chaboyer, B. 1994, in ESO Conf. and Workshop Proc. 49, Dwarf Galaxies, ed. G. Meylan \& P. Prugniel (Haute Provence: ESO), 485

Covino, S., Galletti, S., \& Pasinetti, L. E. 1995, A\&A, 303, 79

da Costa, G. S. 1991, in IAU Symp. 149, The Stellar Populations of Galaxies, ed. B. Barbuy \& A. Renzini (Dordrecht: Kluwer), 191

Davies, R. L., Sadler, E. M., \& Peletier, R. F. 1993, MNRAS, 262, 650 (DSP) Dekel, A., \& Silk, J. 1986, ApJ, 303, 39

Ferguson, H. C. 1994, in ESO Conf. and Workshop Proc. 49, Dwarf Galaxies,

ed. G. Meylan \& P. Prugniel (Haute Provence: ESO), 475

Ferguson, H. C., \& Binggeli, B. 1994, A\&A Rev., 6, 67
Fisher, D., Franx, M., \& Illingworth, G. 1995, ApJ, 448, 119

González, J. J. 1993, Ph.D. thesis, Univ. of California, Santa Cruz (G93)

González, J. J., \& Gorgas, J. 1997, in preparation

Gorgas, J., Efstathiou, G., \& Aragón-Salamanca, A. 1990, MNRAS, 245, 217

Gorgas, J., Faber, S. M., Burstein, D., González, J. J., Courteau, S., \& Prosser, C. 1993, ApJS, 86, 153

Gorgas, J., Pedraz, S., Guzmán, R., González, J. J., \& Cardiel, N. 1997, in preparation

Gregg, M. D. 1991, in IAU Symp. 149, The Stellar Populations of Galaxies, ed.

B. Barbuy \& A. Renzini (Dordrecht: Kluwer), 426

Guzmán, R., Lucey, J. R., \& Bower, R. G. 1993, MNRAS, 265, 731

Held, E. V., \& Mould, J. R. 1994, AJ, 107, 1307

Kormendy, J. 1985, ApJ, 295, 73

Kormendy, J., \& Bender, R. 1994, in ESO Conf. and Workshop Proc. 49, Dwarf

Galaxies, ed. G. Meylan \& P. Prugniel (Haute Provence: ESO), 161

Peletier, R. F. 1989, Ph.D. thesis, Rijksuniversiteit Groningen

Salpeter, E. E. 1955, ApJ, 121, 161

Vader, J. P., Vigroux, L., Lachièze-Rey, M., \& Souviron, J. 1988, A\&A, 203, 217

Vazdekis, A., Casuso, E., Peletier, R. F., \& Beckman, J. E. 1996, ApJS, 106, 307

Worthey, G. 1994, ApJS, 95, 107

Worthey, G., Faber, S. M., \& González, J. J. 1992, ApJ, 398, 69 